\title{
A SIMULATION OF RECRYSTALLIZATION TEXTURES OF Al-ALLOYS WITH CONSIDERATION OF THE PROBABILITIES OF NUCLEATION AND GROWTH
}

\author{
O. ENGLER \\ Los Alamos National Laboratory, Center for Materials Science, K765, \\ Los Alamos, NM 87545, USA
}

(Received in final form 28 September 1997)

The characteristic recrystallization texture components of cold rolled Al-alloys can be traced back to a growth selection of grains with an approximate $40^{\circ}\langle 111\rangle$ orientation relationship out of a limited spectrum of preferentially formed nucleus orientations. Accordingly, recrystallization textures can be modeled by the multiplication of a function $f(g)^{\text {nucl }}$ describing the probability of nucleation of the various orientations and a function $f(g)^{\text {grow }}$ representing their growth probability.

Whereas the growth probability can be accounted for by a $40^{\circ}\langle 111\rangle$ transformation of the rolling texture, the nucleation probability of the respective grains is given by the distribution of potential nucleus orientations, which is known from local texture analysis for the most important nucleation sites in cold rolled Al-alloys, cube-bands, grain boundaries and second-phase particles. If several nucleation sites are active simultaneously, the nucleation probabilities have to be weighted according to their respective proportions. For that purpose, the numbers of nuclei forming at the various nucleation sites were calculated according to a model approach proposed by Vatne et al. (Acta Mater. 44, 1996, 4463-4473).

The paper describes the model for recrystallization texture simulation in Al-alloys and gives examples of recrystallization textures simulated regarding a variation of different microstructural parameters to demonstrate the predictive power of the model.

Keywords: Recrystallization texture simulation; Transformation texture;

Recrystallization; Oriented nucleation; Growth selection; Al-alloys; Cube-bands;

PSN; Grain boundaries

\section{INTRODUCTION}

Recrystallization of deformed metallic materials proceeds by the formation of new undeformed grains in the as-deformed microstructure, 
their so-called 'nucleation', and their subsequent growth into the deformed microstructure. As these mechanisms are characterized by the formation and motion of high-angle grain boundaries, recrystallization generally leads to a change in the crystallographic texture. As an example, Fig. 1(a) and (b) respectively show the orientation distribution functions (ODF) of cold rolled and recrystallization annealed commercial purity A1 (AA1145), as obtained by conventional X-ray texture analysis (Engler et al., 1996a). The ODFs are presented in sections through the three-dimensional orientation space defined by the Euler angles $\varphi_{1}, \Phi, \varphi_{2}$ (Bunge, 1982). Whereas the deformation texture (Fig. 1(a)) is composed of the typical $\beta$-fiber with the well known rolling texture components $\mathrm{C}=\{112\}\langle 111\rangle, \mathrm{S}=\{123\}\langle 634\rangle$ and $\mathrm{B}=$ $\{011\}\langle 211\rangle$, the recrystallization texture (Fig. 1(b)) comprises the cubeorientation $\{001\}\langle 100\rangle$ and an orientation close to the rolling texture $\mathrm{S}$-orientation, which is commonly referred to as R-orientation.

In deformed Al-alloys nucleation of recrystallization generally takes place by enhanced subgrain growth in the vicinity of structural heterogeneities, where substantially larger local misorientations exist than in the homogeneously deformed matrix (Doherty, 1978). In commercially rolled $\mathrm{Al}$-alloys, mainly three nucleation sites are of importance, namely

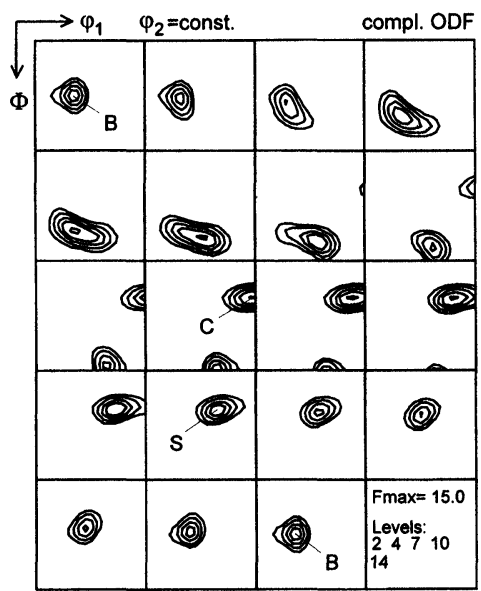

(a) $95 \%$ red., as-rolled

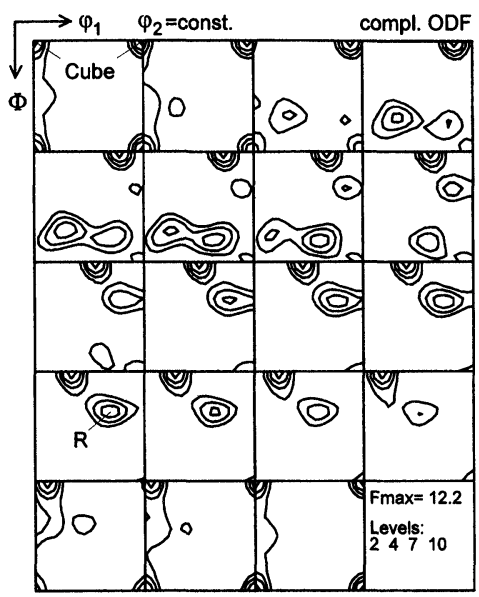

(b) $95 \%$ red., annealed for $1000 \mathrm{~s}$ at $350^{\circ} \mathrm{C}$

FIGURE 1 Rolling and recrystallization texture of commercial purity A1 (AA1145). 
cube-bands, grain boundaries and large second-phase particles (e.g. Juul Jensen et al., 1985; Hjelen et al., 1991; Weiland et al., 1994; Engler, 1996). By means of local texture analysis the occurrence of oriented nucleation in the sense of the preferred formation of special orientations at each of these characteristic nucleation sites could definitely be proved, and for each a specific 'nucleation spectrum', or a characteristic nucleus orientation distribution, has been identified.

With regard to the growth of the new grains, it has often been shown that the orientations obtained after recrystallization of Al-alloys can be related to the rolling texture by a $40^{\circ}\langle 111\rangle$ rotation (e.g. Lücke and Engler, 1990). Detailed local texture analysis of the new recrystallizing grains nucleating at the cube-bands and the grain boundaries by means of electron backscattering diffraction (EBSD) indeed substantiated a growth selection of the cube- and the R-orientations out of the respective nucleus spectra (Juul Jensen, 1995; Engler, 1998) - i.e. exactly of those orientations which have an approximate $40^{\circ}\langle 111\rangle$ orientation relationship to the rolling texture - although the underlying reason is not completely understood so far. For grains nucleating at large constituent particles, in contrast, a preference of $40^{\circ}\langle 111\rangle$ oriented grains at the very early stages of recrystallization, but no further selection during later growth states, has been observed, which is called micro-growth selection (Engler et al., 1996b).

In conclusion, the recrystallization textures of $\mathrm{Al}$-alloys evolve by a preferred formation of some orientations at characteristic nucleation sites and a subsequent growth selection of distinct orientations out of this spectrum of nucleus orientations (Engler, 1996). In a preceding paper (Engler, 1997a) it has been demonstrated that in such a case the recrystallization textures can be successfully modeled by multiplication of a function $f(g)^{\text {nucl }}$ that represents the probability of the nucleation of the new grains with their growth probability function $f(g)^{\text {grow }}$ (Fig. 2).

The probability of nucleation is given by the distribution of potential nucleus orientations, i.e. the orientation spectra of the corresponding nucleation sites. In the foregoing paper, ODFs calculated from EBSDdata obtained at the various nucleation sites were used as the respective nucleation probability functions $f(g)^{\text {nucl }}$. In most Al-alloys, nucleation is not restricted solely to one nucleation site, but nuclei form simultaneously at various structural heterogeneities in the microstructure. The recrystallization textures then result from a competition between 


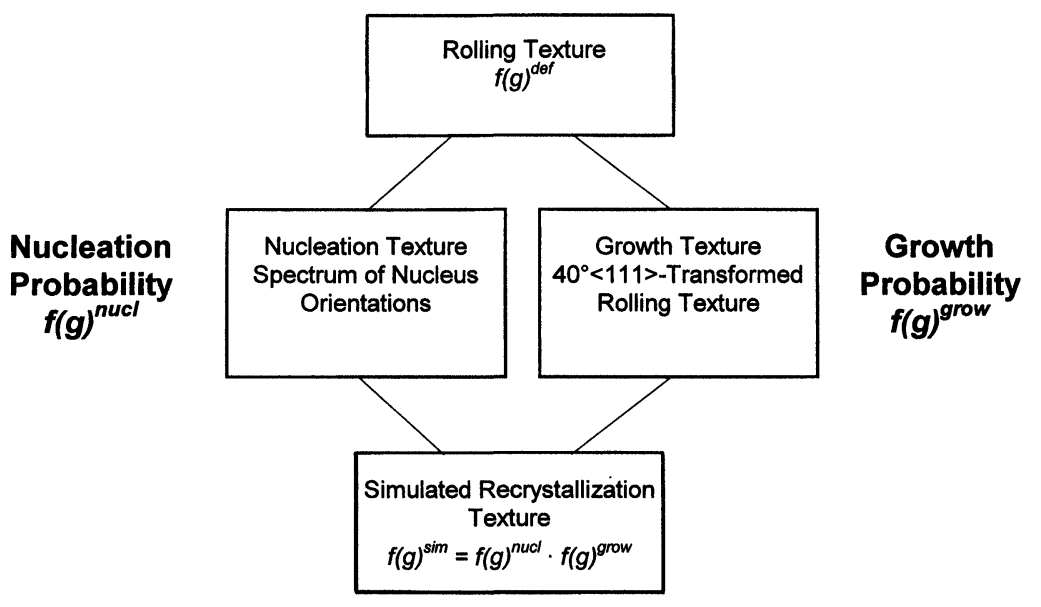

FIGURE 2 Scheme of the model.

the corresponding orientations in dependence on the number and efficiency of the various nucleation sites. In the present model, this is taken into account by a weighted overlapping of the respective nucleus orientation distributions according to:

$$
f(g)^{\mathrm{nucl}}=x_{\mathrm{Cube}} \cdot f(g)_{\mathrm{Cube}}^{\mathrm{nucl}}+x_{\mathrm{GB}} \cdot f(g)_{\mathrm{GB}}^{\mathrm{nucl}}+x_{\mathrm{PSN}} \cdot f(g)_{\mathrm{PSN}}^{\mathrm{nucl}} .
$$

The weight factors $x_{i}$ denote the corresponding probability or efficiency of nucleation (with $x_{\text {Cube }}+x_{\mathrm{GB}}+x_{\mathrm{PSN}}=1$ ) and the functions $f(g)_{\text {Cube }}^{\text {nucl }}$, $f(g)_{\mathrm{GB}}^{\text {nucl }}$ and $f(g)_{\mathrm{PSN}}^{\text {nucl }}$ denote the orientation spectra of the cube-bands, grain boundaries and particles, respectively.

The growth probability function $f(g)^{\text {grow }}$ is derived from a $40^{\circ}\langle 111\rangle$ transformation of the corresponding rolling texture $f(g)^{\text {def }}$ to consider the $40^{\circ}\langle 111\rangle$ (micro)growth selection. As an example, Fig. 3 shows the $40^{\circ}\langle 111\rangle$ transformed rolling texture $f(g)^{\text {trans }}$ of $95 \%$ cold rolled AA 1145 (see Fig. 1(a)).

To superimpose both the nucleation and the growth probability and, thus, to obtain the probability of an orientation arising in the simulated recrystallization texture $f(g)^{\text {sim }}$, finally the $f(g)$-values of the ODFs $f(g)^{\text {nucl }}$ and $f(g)^{\text {grow }}$ are multiplied (Fig. 2):

$$
f(g)^{\text {sim }}=f(g)^{\text {nucl }} \cdot f(g)^{\text {grow }} .
$$




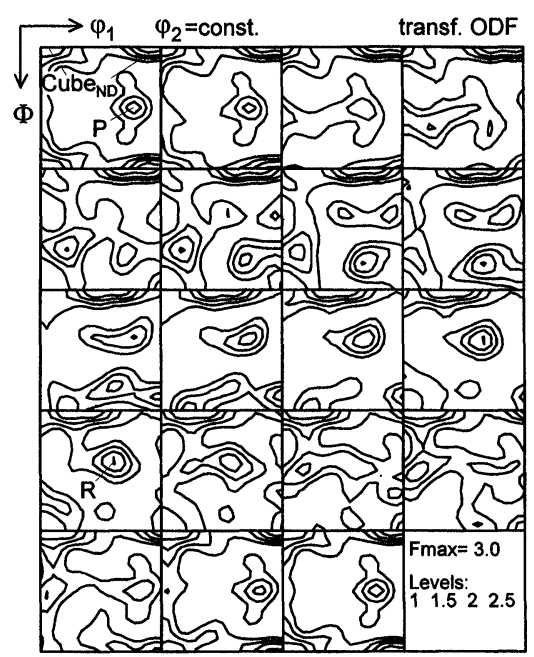

FIGURE $340^{\circ}\langle 111\rangle$ transformed rolling texture of AA1145 (95\% red.).

Sometimes, truncation errors during the series expansion cause negative values in one of the ODFs. As these are physically meaningless, they are set to zero, so that $f(g)^{\text {sim }}$ has to be re-normalized, although the resulting normalization factors typically are very small $(<1.2)$. Finally, the ODFs can be plotted and compared to the corresponding experimental recrystallization textures.

The obtained model results supported the assumption of a $40^{\circ}\langle 111\rangle$ (micro)growth selection out of a limited spectrum of preferentially formed nucleus orientations (Engler, 1997a). Moreover, the model as it stands provides valuable information on the impact of a varying efficiency of nucleation at the different sites on the final recrystallization textures. However, detailed local texture analysis of the potential nucleus orientations is of course not feasible for large-scale texture simulations, in particular with view to the ultimate aim of predicting the recrystallization textures of commercial materials for a wide range of different processing parameters. Therefore, based on the EBSD-results, for either nucleation site a nucleus orientation distribution function $f(g)_{\text {Cube }}^{\text {nucl }}, f(g)_{\mathrm{GB}}^{\text {nucl }}$ and $f(g)_{\mathrm{PSN}}^{\text {nucl }}$ was generated. The weight factors $x_{i}$ in Eq. (1) were derived according to a recent approach to calculate the number of nuclei forming at each nucleation site (Vatne et al., 1996). 
In the present paper, the basic ideas of the model are outlined and, subsequently, simulation results obtained with a variation of different microstructural parameters - strain, particle distribution and initial grain size - are presented which demonstrate the predictive power of the model. Model predictions pertaining to the recrystallization of hot deformed Al-alloys will be presented in a separate paper (Engler and Vatne, 1998).

\section{DESCRIPTION OF THE MODEL}

\subsection{Generation of the Nucleus Orientation Distribution Functions $f(g)^{\text {nucl }}$}

The recrystallization textures of most Al-alloys are dominated by a strong cube-orientation $\{001\}\langle 100\rangle$ with pronounced scatter about the rolling direction (RD) towards Goss $=\{011\}\langle 100\rangle$ (e.g. Fig. 1(b)). Nucleation of cube-oriented grains is known to take place in the socalled cube-bands, band-like structures that are part of the as-deformed microstructure (e.g. Hjelen et al., 1991; Weiland and Hirsch, 1991; Doherty et al., 1995). Detailed local texture analysis of the subgrains within the cube-bands as well as the grains that evolve out of the cubebands has shown that the cube-bands comprise strong rotations from the exact cube-orientation either around the RD or, less pronounced, about the transverse direction (TD) (Engler et al., 1996b). Accordingly, an orientation spectrum for nuclei forming in cube-bands, i.e. the nucleus orientation distribution $f(g)_{\text {Cube }}^{\text {nucl }}$, has been synthesized which consists of the cube-orientation and its RD- and TD-rotations. Although the resulting ODF (Fig. 4) is quite sharp $\left(f(g)_{\max }>20\right)$, it turned out to yield satisfactory results for a wide range of model predictions.

Besides the cube-bands, the large-angle grain boundaries between the deformed grains or bands can act as viable nucleation sites. Local texture analysis of new grains that have nucleated at such grain boundaries showed that this mechanism - which is commonly referred to as strain induced boundary migration (SIBM) - leads to the formation of grains with orientations close to those present in the deformation texture (Bellier and Doherty, 1977). An ODF as computed from a multitude of such SIBM-grains strongly resembled the rolling texture $f(g)^{\text {def }}$ of the 


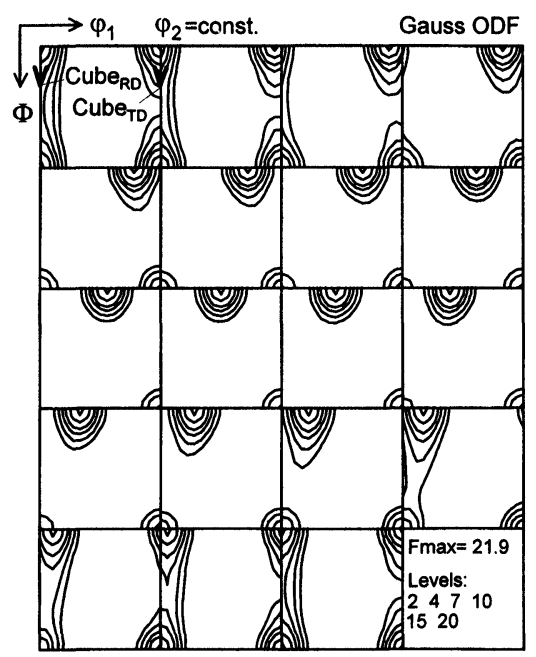

FIGURE 4 Synthesized spectrum of nucleus orientations within the cube-bands $f(g)_{\text {Cube }}^{\text {nucl }}$.

given sample, but it revealed larger orientation scatter which apparently reflects the orientation gradients in the vicinity of the grain boundaries (Engler, 1996; 1998). Accordingly, the nucleus spectrum $f(g)_{\mathrm{GB}}^{\text {nucl }}$ was derived by an artificial weakening (randomizing) of the respective rolling texture according to:

$$
f(g)_{\mathrm{GB}}^{\mathrm{nucl}}=\frac{x_{\mathrm{GB}}}{2} \cdot f(g)^{\mathrm{def}}+\frac{x_{\mathrm{GB}}}{2} \cdot 1 .
$$

Particle stimulated nucleation (PSN) takes place in the deformation zones that form during deformation around large particles to accommodate deformation incompatibilities (Humphreys, 1977). In polycrystalline materials mostly a random orientation distribution of the deformation zones is reported (e.g. Humphreys, 1977; Oscarsson, 1991), which means that the nucleation probability $f(g)_{\mathrm{PSN}}^{\text {nucl }}$ would be 1 . Accordingly, PSN-recrystallization textures have quite successfully been simulated by a simple $40^{\circ}\langle 111\rangle$-transformation of the corresponding rolling texture to consider the micro-growth selection (Engler, 1997a). However, the modeled textures always depicted some characteristic deviations, and detailed local texture analysis indeed proved that the orientation distributions of the subgrains in the deformation 
zones and, consequently, the potential nuclei are not entirely random (Engler et al., 1997). Rather, the orientations within the deformation zones can be deduced from the corresponding matrix orientations by an approximate $\langle 112\rangle$-rotation, which is attributed to the particle rotation caused by the active slip systems (Russell and Ashby, 1970; Humphreys, 1979; Engler, 1996). Hence, in order to model the spectrum of available nucleus orientations it appeared reasonable to perform a $\langle 112\rangle$ transformation of the rolling texture. However, a simulation of the rolling texture evolution proved that the $\langle 112\rangle$-roller axes are not randomly distributed but show a strong concentration close to the TD (Engler, 1997b). Therefore, the contribution of each $\langle 112\rangle$-axis $\vec{r}_{112}$ is weighted with the term $\cos \left(\vec{r}_{112} \wedge T D\right)$ by its angle to the TD; the resulting weight factors as plotted in the stereographic projection are shown in Fig. 5(a). With regard to the rotation angle, it turned out that a rotation by $35^{\circ}$ gives best results. Figure 5(b) shows the ODF representing the PSN-nucleus spectrum of AA1145 according to this weighted $35^{\circ}\langle 112\rangle$-transformation. The texture is very weak, pointing to the near-random orientation spectrum of PSN-nuclei. However, it comprises some intensities of a cube-orientation that is rotated about the normal direction (ND) and of the P-orientation $\{011\}\langle 122\rangle$, which means that these orientation will be enhanced in the modeled recrystallization textures (see below).

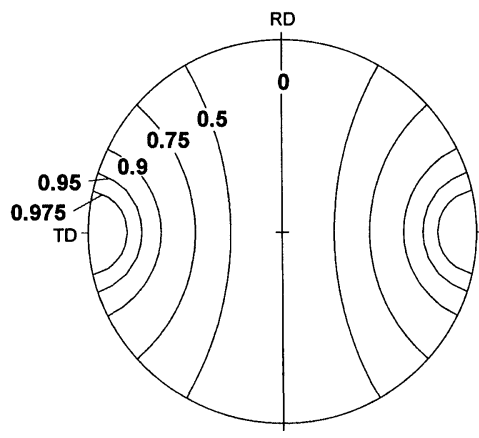

(a) weight factors of the $<112>$-axes

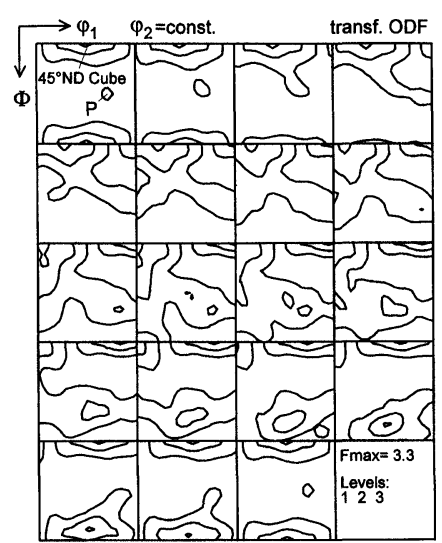

(b) $35^{\circ}<112>$-transformed rolling texture

FIGURE 5 PSN-nucleus spectrum $f(g)_{\mathrm{PSN}}^{\text {nucl }}$ (AA1145, 95\% red.). 
With these three nucleus orientation distributions, as well as the corresponding $40^{\circ}\langle 111\rangle$-transformed rolling texture to consider the growth selection, the recrystallization texture of commercial purity $\mathrm{A} 1$ (AA1145) shown in Fig. 1(b) was simulated. The resulting texture obtained with the weight factors $x_{\mathrm{Cube}}=0.2, x_{\mathrm{GB}}=0.7$ and $x_{\mathrm{PSN}}=0.1-$ is shown in Fig. 6. Comparison with the experimental recrystallization texture (Fig. 1(b)) proves the very good agreement in both the intensity and the position of the main recrystallization texture components.

\subsection{Determination of the Weight Factors $x_{i}$}

As demonstrated in the above example, the present model allows one to readily simulate recrystallization textures and, in particular, it is possible to study the contribution of the different nucleation sites to the final recrystallization textures. However, the determination of the weight factors $x_{i}$ was purely arbitrary so far, which prevented meaningful texture predictions. Therefore, to enable the model to simulate recrystallization textures over a wider range of experimental parameters, a suitable approach to determine the weight factors $x_{i}$ was required. Recently, Vatne et al. (1996) developed a microstructurally sound

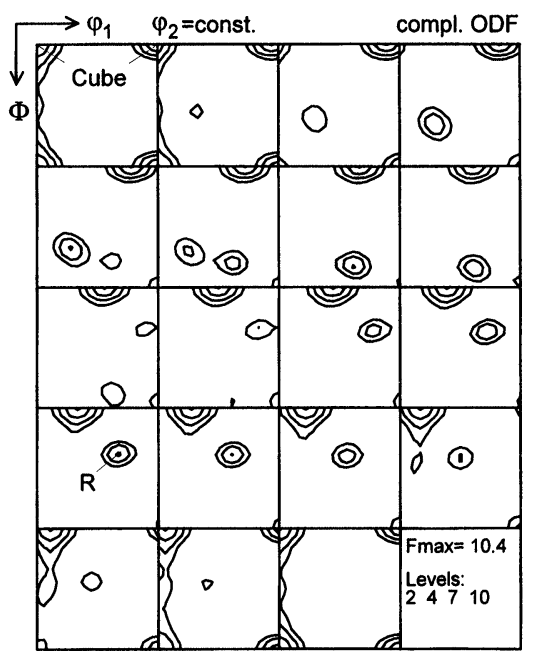

FIGURE 6 Modeled recrystallization texture of AA1145 (95\% red.; see Fig. 1(b)). 
recrystallization model which yields the number of nuclei $N_{\text {Cube }}, N_{\text {PSN }}$ and $N_{\mathrm{GB}}$ forming at the respective nucleation sites. This approach, which is based on experimental investigations on the nature and particularly on the evolution of the various nucleation sites during rolling deformation, has been adapted with minor changes so as to yield the weight factors $x_{i}$ necessary to simulate recrystallization textures in the present model. In the following, the principles to derive $N_{\text {Cube }}, N_{\text {PSN }}$ and $N_{\mathrm{GB}}$ are briefly outlined, as far as they are required for the present model; for more details the reader is referred to the original paper by Vatne et al. (1996).

PSN takes place by accelerated subgrain growth within the deformation zones around the particles and subsequent growth out of the deformation zones into the surrounding matrix (Humphreys, 1977). As the maximum possible nucleus size is limited to the size of the deformation zones $\lambda$, which again is linked to the particle size $\eta$, a critical particle size $\eta^{*}$ for successful nucleation can be expressed as:

$$
\eta^{*} \approx \frac{\lambda}{2}=\frac{1}{2} \cdot \frac{4 \cdot \gamma_{\mathrm{GB}}}{p_{\mathrm{D}}} .
$$

In this relation, $\gamma_{\mathrm{GB}}$ indicates the specific grain boundary energy, which was chosen to $0.3 \mathrm{~J} / \mathrm{m}^{2}$ throughout the present calculations, and $p_{\mathrm{D}}$ is the driving force, which will be addressed later. In the case of PSN at particles with a size distribution $f(\eta)$, the density of PSN-sites $N_{\text {PSN }}$ becomes:

$$
N_{\mathrm{PSN}}=c_{\mathrm{PSN}} \int_{\eta^{*}}^{\infty} f(\eta) \mathrm{d} \eta
$$

( $c_{\mathrm{PSN}}$ : modeling constant describing the efficiency of PSN; see below). In most Al-alloys, the distribution of large particles can be represented in terms of the following relationship (Nes and Hutchinson, 1989):

$$
f(\eta)=H \cdot \exp (-L \eta)
$$

with the characteristic distribution parameters $H$ and $L$. Combining Eqs. (4)-(6) and setting $N_{0}=H / L$ yields a density of PSN-nuclei:

$$
N_{\mathrm{PSN}}=c_{\mathrm{PSN}} N_{0} \exp \left(-\frac{2 L \gamma_{\mathrm{GB}}}{p_{\mathrm{D}}}\right)
$$


Thus, besides the characteristic particle distribution parameters $N_{0}$ (or $H)$ and $L, N_{\mathrm{PSN}}$ mainly depends on the driving force for recrystallization $p_{\mathrm{D}}$, which can be determined as follows. In deformed, and in particular in briefly recovery-annealed, Al-alloys the driving force is composed of two contributions, the energy stored in the subgrain boundaries and the energy of the dislocations in the subgrain interior:

$$
p_{\mathrm{D}}=\frac{\alpha \gamma_{\mathrm{SB}}}{\delta}+\rho \Gamma
$$

with $\alpha$ : geometric constant of the order of $3, \gamma_{\mathrm{SB}}$ : specific subgrain boundary energy, $\Gamma$ : dislocation line tension which is usually estimated by $0.5 \cdot \mu b^{2}$ ( $\mu$ and $b$ respectively are the shear modulus $(27 \mathrm{GPa}$ ) and the Burgers-vector $(0.286 \mathrm{~nm})$ of $\mathrm{Al})$. The dislocation density within the subgrains, $\rho$, and the average subgrain size, $\delta$, have been found to be linked through the relation:

$$
\sqrt{\rho}=C / \delta
$$

( $C$ : constant of the order of 2). The specific subgrain boundary energy $\gamma_{\mathbf{S B}}$ can be expressed in terms of the well-known Read-Shockleyrelation:

$$
\gamma_{\mathrm{SB}}=\frac{\mu b \theta}{4 \pi(1-\nu)}\left(1+\ln \frac{b}{2 \pi r_{0} \theta}\right)
$$

( $\nu$ : Poisson-ratio $(0.33), r_{0}$ : radius of the dislocation core). $\theta$ is the average misorientation between neighboring subgrains, and for the evolution of $\theta$ with strain $\varepsilon$ the following empirical relation has been used:

$$
\theta=3^{\circ}-\exp (-3 \varepsilon)
$$

Throughout the present calculations, the logarithmic strain $\varepsilon=\ln \left(t_{0} / t\right)$ has been used, where $t_{0}$ and $t$ respectively denote the initial and the final thickness of the rolled sheets. The subgrain boundary energy $\gamma_{\mathrm{SB}}$ can be linked to the energy of an ordinary grain boundary, $\gamma_{\mathrm{GB}}$, by inserting the maximum angle of a low-angle grain boundary $\theta_{\mathrm{c}}$ in the ReadShockley-relation, Eq. (10) (with $\theta_{\mathrm{c}}=15^{\circ}$ ). Thus, the driving force $p_{\mathrm{D}}$ 
can be written as:

$$
p_{\mathrm{D}}=\frac{\alpha \gamma_{\mathrm{GB}} \theta}{\delta \theta_{\mathrm{c}}} \ln \left(\frac{e \theta_{\mathrm{c}}}{\theta}\right)+\frac{C^{2} \mu b^{2}}{2 \delta^{2}} .
$$

To calculate the driving force for recrystallization $p_{\mathrm{D}}$, the average subgrain size $\delta$ must be known. After cold deformation, the evolution of $\delta$ with strain $\varepsilon$ was computed according to the following empirical relation:

$$
\delta=3.5 \cdot 10^{-7} \mathrm{~m}+1.7 \cdot 10^{-7} \mathrm{~m} / \varepsilon .
$$

As an example, for $\varepsilon=1$ and 3 Eq. (13) yields an average subgrain size $\delta=0.52 \mu \mathrm{m}$ and $0.41 \mu \mathrm{m}$, respectively, which is in good agreement with experimental results. After hot deformation, additionally the influence of deformation temperature and strain rate on the subgrain size has to be considered, as discussed in detail by Vatne et al. (1996).

As already mentioned above, nucleation of cube-oriented grains takes place in cube-bands, band-like structures which pre-exist in the asdeformed microstructure. The number of cube-nuclei $N_{\text {Cube }}$ is given by the volume of cube-orientation $M_{\text {Cube }}$ within these cube-bands, the boundary area $A$ between cube-bands and surrounding matrix, and by the fraction of cube-subgrains $S_{\text {Cube }}^{*}$ in the cube-bands which exceed the critical size for nucleation $\delta^{*}$ :

$$
N_{\text {Cube }}=c_{\text {Cube }} \frac{A(\varepsilon) M_{\text {Cube }} S_{\text {Cube }}^{*}}{\delta_{\text {Cube }}^{2}}
$$

( $c_{\text {Cube }}$ : modeling constant describing the efficiency of nucleation at cubebands). $\delta_{\text {Cube }}$ is the average subgrain size within the cube-bands which in accordance with detailed experimental observations - has been assumed to be 1.5 times larger than the subgrain size in the matrix $\delta$.

The evolution of the volume of cube-orientation within the cubebands $M_{\text {Cube }}$ starting from the initial volume fraction $M_{\text {Cube }}^{0}$ with strain $\varepsilon$ is determined from a normalized curve that was derived from a variety of experimental data (Fig. 7) according to:

$$
M_{\text {Cube }}=M_{\text {Cube }}^{0} \cdot(1+0.5 \varepsilon)^{-3} \text {. }
$$

With increasing strain $\varepsilon$ the area $A$ between the cube-bands and the surrounding matrix increases, and from simple geometrical 


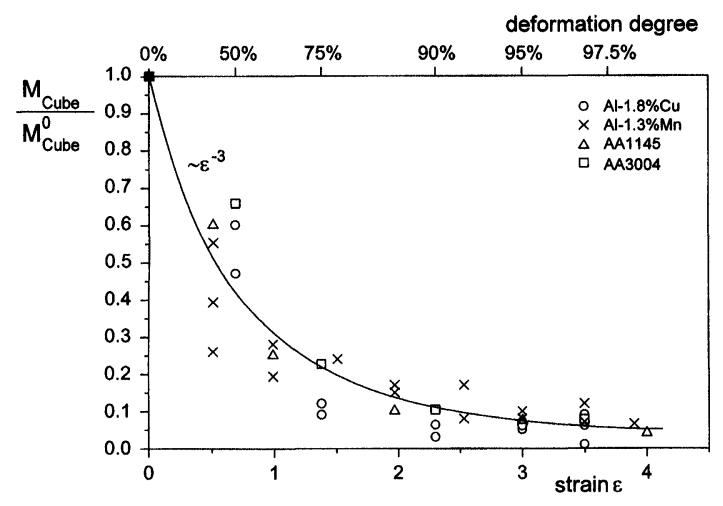

FIGURE 7 Evolution of the volume fraction $M$ of the cube-orientation with strain $\varepsilon$.

considerations it follows:

$$
A(\varepsilon)=2 \cdot[\exp (\varepsilon)+\exp (-\varepsilon)+1] / D_{0}
$$

$\left(D_{0}\right.$ : initial grain size). The number $S_{\text {Cube }}^{*}$ of overcritically large cubesubgrains with size exceeding $\delta^{*}$ can be derived from integration of the subgrain size distribution function $f_{\text {Cube }}(\delta)$ :

$$
S_{\text {Cube }}^{*}=\int_{\delta^{*}}^{\infty} f_{\text {Cube }}(\delta) \mathrm{d} \delta .
$$

Vatne et al. (1996) fitted the subgrain size distributions of both cubesubgrains and subgrains in the deformed matrix in various Al-alloys with a $\Gamma$-distribution function, which allows an analytical solution of the integral in Eq. (17). With $\delta^{*}=4 \gamma_{\mathrm{GB}} / p_{\mathrm{D}}$ (see Eq. (4)) it follows:

$$
S_{\text {Cube }}^{*}=\exp (-a)\left[1+a+\frac{1}{2 !}(a)^{2}+\frac{1}{3 !}(a)^{3}+\frac{1}{4 !}(a)^{4}\right], \quad a=\frac{5 \delta^{*}}{\bar{\delta}_{\text {Cube }}} .
$$

For nucleation at grain boundaries, analogous considerations yield a density of nucleation sites $N_{\mathrm{GB}}$ of:

$$
N_{\mathrm{GB}}=c_{\mathrm{GB}} \frac{A(\varepsilon)\left(1-M_{\mathrm{Cube}}\right) S_{\mathrm{GB}}^{*}}{\delta^{2}}
$$


( $c_{\mathrm{GB}}$ : modeling constant describing the efficiency of nucleation at grain boundaries).

With these numbers of nucleation sites $N_{i}$, finally the weight factors $x_{i}$ can be determined:

$$
x_{i}=\frac{N_{i}}{N_{\mathrm{PSN}}+N_{\text {Cube }}+N_{\mathrm{GB}}} .
$$

Besides the recrystallization texture, the final recrystallized grain size $\mathrm{D}_{\mathrm{RX}}$ can be estimated by:

$$
D_{\mathrm{RX}}=\frac{G}{\left(N_{\mathrm{PSN}}+N_{\text {Cube }}+N_{\mathrm{GB}}\right)^{1 / 3}}
$$

( $G$ : modeling constant representing a growth efficiency).

\section{APPLICATION OF THE MODEL}

\subsection{Influence of the Degree of Deformation}

To assess the capability of the model of predicting recrystallization textures, the recrystallization textures of AA1145 cold rolled to various reductions (strains $\varepsilon=1,2,3$ and 4 ) have been simulated; one example of this series has already been shown above (Fig. 6). The necessary input data for $M_{\text {Cube }}^{0}, D_{0}$ and the particle size distribution parameters $N_{0}$ and $L$ were taken from an earlier experimental paper (Engler et al., 1996a), the rolling and recrystallization textures obtained in AA1145 can also be found in that paper.

The modeling constants $c_{i}$ were derived by fitting the experimental recrystallization texture for $\varepsilon=3$ (Fig. 1(b)) to $c_{\text {Cube }}=1.0, c_{\mathrm{GB}}=0.8$ and $c_{\mathrm{PSN}}=0.15$; the other simulations were then all performed with the same constants $c_{i}$. Figure 8(a) shows the evolution of the weight factors $x_{i}$ with increasing $\varepsilon$. It turned out that $x_{\mathrm{GB}}$ strongly increased from about 0 to values exceeding 0.8 , reflecting the strong increase in grain boundary area $A(\varepsilon)$ (Eq. (16)). Simultaneously, $x_{\text {Cube }}$ and $x_{\text {PSN }}$ decreased, although the corresponding nucleation site numbers, $N_{\text {Cube }}$ and $N_{\mathrm{PSN}}$, happened to increase slightly. This strong increase in the total number of nucleation sites $N_{i}$ gave rise to the strong decrease in the final recrystallized grain size $D_{\mathrm{RX}}$ (Eq. (21)) with strain, which is in very good agreement with the experimental data (Engler et al., 1996a). 


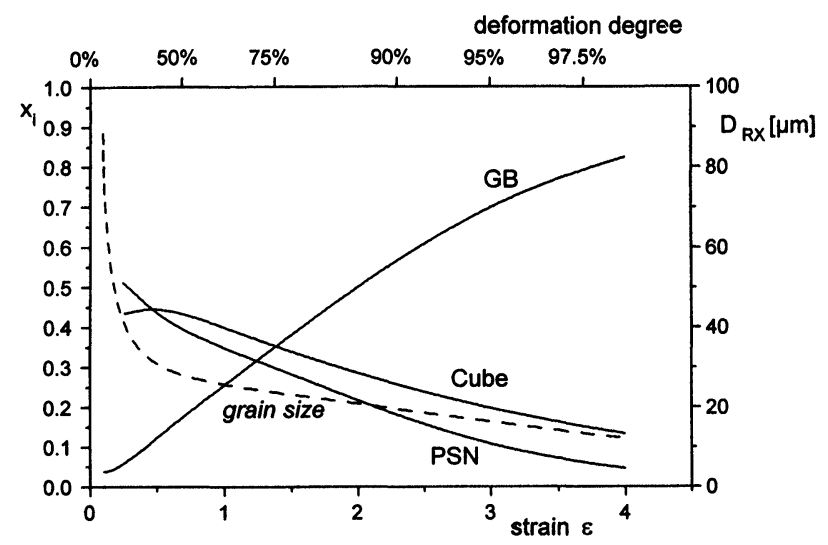

(a) evolution of the weight factors $x_{i}$

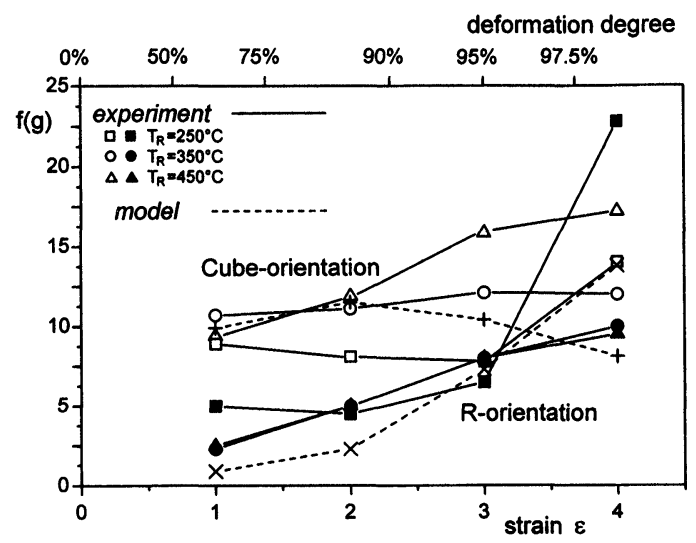

(b) comparison of model and experimental results

FIGURE 8 Modeling of the recrystallization behavior of AA1145 in dependence on the strain $\varepsilon$.

The large resemblance between the simulated and the experimental recrystallization textures has already been demonstrated in Fig. 6. Here however, instead of representing the entire ODFs for all cases, the data were condensed to show the maximum intensity $f(g)_{\max }$ of the characteristic recrystallization texture orientations, i.e. the cube- and the R-orientation (Fig. 8(b)). It appears that the model is capable of 
predicting the main tendency of recrystallization texture evolution, even in a quantitative manner. In particular the strong increase of the $\mathrm{R}$-orientation and the more or less constant intensities of the cubeorientation are correctly predicted. However, this example also visualizes one of the major drawbacks of the present model. Whereas the experimental results clearly proved an impact of the annealing temperature (Fig. 8(b)) - which is discussed in detail by Engler et al. (1996a) no such influence is considered in the model calculations so far.

\subsection{Influence of the Initial Grain Size}

The influence of the grain size prior to deformation on the eventually resulting recrystallization textures has been studied in the two-phase alloy $\mathrm{Al}-1.8 \% \mathrm{Cu}$ (Engler, 1995). It turned out that an increase in the initial grain size $D_{0}$ led to a substantial shift from a cube-recrystallization texture towards a texture which is controlled by orientations that are typically attributed to PSN, i.e. the ND-rotated cube-orientation $\{001\}\langle 310\rangle$ and the P-orientation $\{011\}\langle 122\rangle$. As an example, the texture of an initially fine grained sample after $90 \%$ rolling reduction and subsequent recrystallization is shown in Fig. 9. With regard to the present model, the initial grain size $D_{0}$ affects nucleation at cube-bands and at grain boundaries by influencing the available area of nucleation $A(\varepsilon)$ (Eq. (16)). An increase in $D_{0}$ results in a decrease in $A(\varepsilon)$ and,

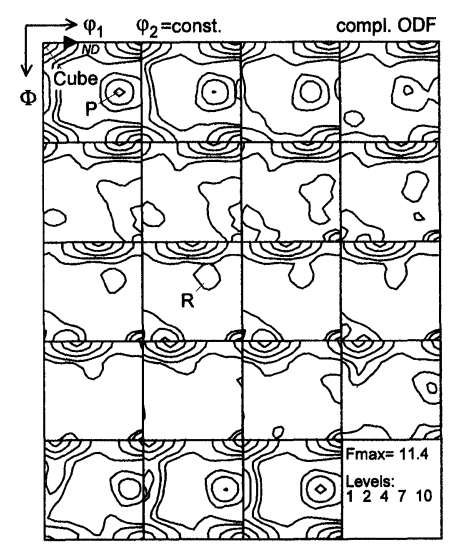

FIGURE 9 Recrystallization texture of fine grained $\mathrm{Al}-1.8 \% \mathrm{Cu}\left(D_{0}=40 \mu \mathrm{m}, 90 \%\right.$ red., recrystallization annealed for $3 \mathrm{~s}$ at $450^{\circ} \mathrm{C}$ ). 
consequently, in less nuclei forming at both cube-bands and grain boundaries. The evolution of the weight factors $x_{i}$ with increasing initial grain size $D_{0}$ was simulated by varying $D_{0}$ for two different strains, $\varepsilon=2.3(90 \%)$ and $\varepsilon=3.7(97.5 \%)$. In this case, simply a constant density of overcritically large particles of $10^{16} \mathrm{~m}^{-3}$ was assumed; the modeling constants $c_{i}$ came out to $c_{\mathrm{PSN}}=1.0, c_{\mathrm{Cube}}=0.9$ and $c_{\mathrm{GB}}=0.1$. Figure 10(a) and (b) shows the evolution of the weight factors $x_{i}$ with increasing initial grain size $D_{0}$ for the two deformation degrees applied. In both diagrams, $x_{\mathrm{PSN}}$ strongly increases at the cost of $x_{\text {Cube }}$ and $x_{\mathrm{GB}}$, reflecting the observed shift from a cube-recrystallization texture to a texture as controlled by PSN. Interestingly, despite the large variation in initial grain size $D_{0}$ by about two orders of magnitude, the modeled recrystallized grain size $D_{\mathrm{RX}}$ increased only little with $D_{0}$, as has also been observed experimentally (Engler, 1995).

As mentioned above, the recrystallization textures comprised the cube-orientation as well as the typical PSN-orientations cube ${ }_{\mathrm{ND}}$ and $\mathrm{P}$ in different proportions. Therefore, to compare the model predictions with the experimental results, in this example the maximum intensities $f(g)$ of the cube-orientation, inclusive of its ND-rotations, and the Porientation are represented (Fig. 10(c) and (d)). Furthermore, the rotation of the cube-orientation about the $\mathrm{ND}$, as characterized by the Euler-angle $\varphi_{1}$, is shown (which is still $0^{\circ}$ in Fig. 9). Again, the simulations predict the main features of the recrystallization texture very satisfactorily, in particular the shift from the cube- to the PSN-recrystallization texture with increasing $D_{0}$ is nicely reproduced.

\subsection{Influence of the Particle State}

In a recent paper the influence of different particle states on the nucleation of recrystallization and the resulting recrystallization textures has been studied in a binary $\mathrm{Al}-1.3 \% \mathrm{Mn}$ alloy (Engler et al., 1996b). Three samples of this alloy were subjected to different annealing treatments so as to precipitate second-phase particles with different sizes and volume fractions. Whereas one sample which was referred to as A0 was deformed in the supersaturated state, another sample, labeled A170, was annealed for $170 \mathrm{~h}$ at $400^{\circ} \mathrm{C}$ to precipitate a large volume of $\mathrm{Al}_{6} \mathrm{Mn}$ particles prior to cold rolling. Sample A48 was annealed for $48 \mathrm{~h}$ at $400^{\circ} \mathrm{C}$, resulting in an intermediate state of particle size and volume. 


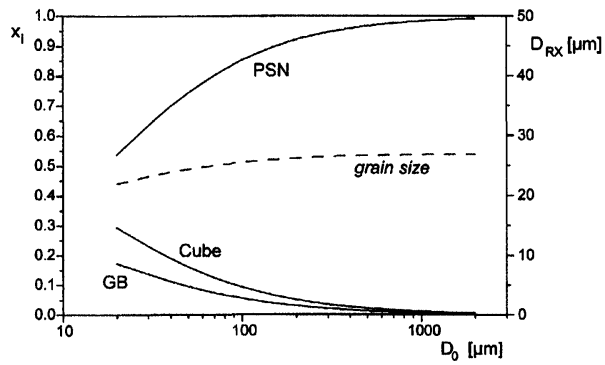

(a) evolution of the weight factors $x_{i}(90 \%$ red.)

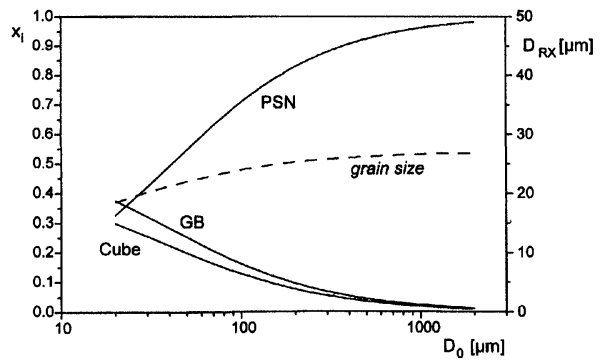

(b) evolution of the weight factors $x_{l}(97.5 \%$ red.)

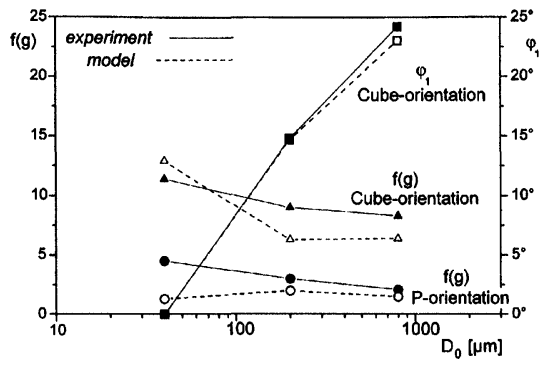

(c) comparison of model and experimental results ( $90 \%$ red.)

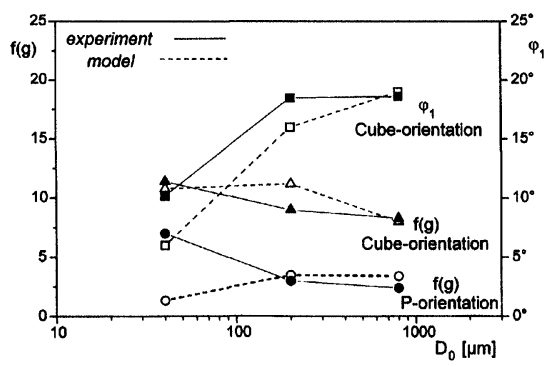

(d) comparison of model and experimental results ( $97.5 \%$ red.)

FIGURE 10 Modeling of the recrystallization behavior of $\mathrm{Al}-1.8 \% \mathrm{Cu}$ in dependence on the initial grain size $D_{0}$. 


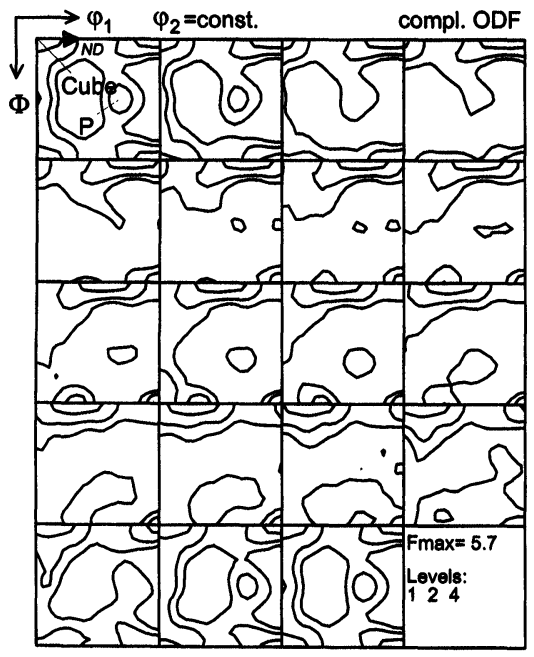

FIGURE 11 Recrystallization texture of $\mathrm{Al}-1.3 \% \mathrm{Mn}$ with largest volume of secondphase particles (sample A170, 97\% red., recrystallization annealed for $3 \mathrm{~s}$ at $450^{\circ} \mathrm{C}$ ).

As an example, Fig. 11 shows the recrystallization texture of sample A170 with largest precipitated volume, which consists of the cubecomponent which indicates nucleation at the cube-bands and strong ND-scatter of the cube-orientation as well as some intensities of the Porientation pointing at PSN. A thorough survey on the rolling and recrystallization textures of these samples can be found in the corresponding experimental paper (Engler et al., 1996b).

In order to study to what extent the present model can account for the influence of the particle state, the recrystallization textures in the three different samples were simulated for various strains $\varepsilon$. Whereas in the supersaturated samples virtually no particles were observed, the particle densities in the aged samples A48 and A170 were estimated to be $N_{0}=3 \cdot 10^{15} \mathrm{~m}^{-3}$ and $N_{0}=10^{16} \mathrm{~m}^{-3}$, respectively, the parameter $L$ was determined to be $0.7 \cdot 10^{6} \mathrm{~m}^{-1}$ in both samples. Besides changing the particle state, the different pre-treatments applied led to different initial grain sizes $D_{0}$ of the three samples, which was also entered in the model. As an example, Fig. 12(a) shows the model predictions for sample A170 in terms of the evolution of the weight factors $x_{i}$ and the grain size $D_{\mathrm{RX}}$ with strain, which were obtained with the modeling constants $c_{\mathrm{PSN}}=1.0$, 


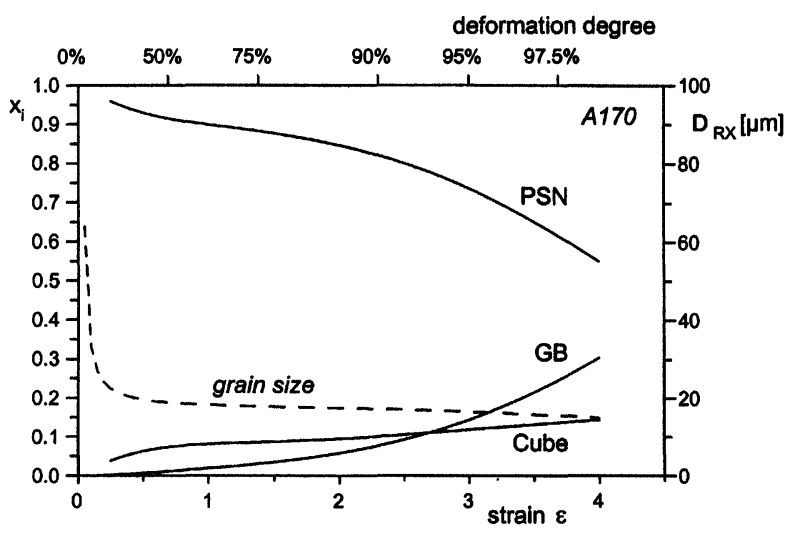

(a) evolution of the weight factors $x_{i}$

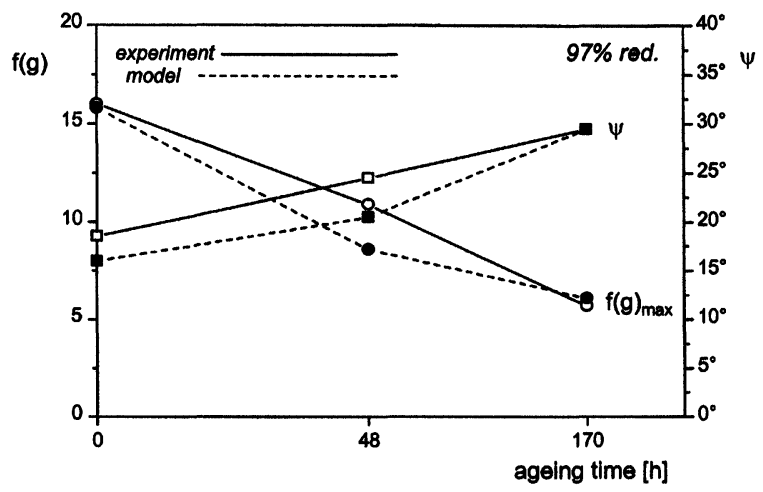

(b) comparison of model and experimental results

FIGURE 12 Modeling of the recrystallization behavior of $\mathrm{Al}-1.3 \% \mathrm{Mn}$ in dependence on the precipitation state.

$c_{\text {Cube }}=0.75$ and $c_{\mathrm{GB}}=0.06$. In this set of experiments, the maximum texture intensity $f(g)_{\max }$ as well as the ND-scatter $\psi$ of the cube-orientation obtained in the model predictions were compared with the experimental results (Fig. 12(b), see Fig. 11). For the latter, the half width of the cube-peak, i.e. the angle $\psi$ at which the cube-peak had decayed to intensity $1 / \mathrm{e}$, was evaluated. As in the previous examples, the model simulates the main features of the recrystallization texture very 
satisfactorily by reproducing the shift from the cube-recrystallization texture towards the PSN-texture as well as the simultaneous texture weakening with increasing precipitated volume $(\mathrm{A} 0 \rightarrow \mathrm{A} 48 \rightarrow \mathrm{A} 170)$. Furthermore, the model predicts a substantial decrease in the final recrystallized grain size - reflecting the increasing efficiency of PSN which has also been observed experimentally (Engler et al., 1996b).

\section{CONCLUSIONS AND OUTLOOK}

Based on the assumption that recrystallization textures in rolled Alalloys evolve by a growth selection of $40^{\circ}\langle 111\rangle$-oriented grains out of a limited spectrum of preferentially formed nucleus orientations, the recrystallization textures can be simulated by the multiplication of the probability of nucleation $f(g)^{\text {nucl }}$ of the orientations with the probability of their growth $f(g)^{\text {grow }}$. In extension of an older model (Engler, 1997a), the number of new grains $N_{i}$ that form at the characteristic nucleation sites, cube-bands, grain boundaries and large second-phase particles, had to be derived to account for the varying contribution of the respective nucleation sites to the final recrystallization textures. For that purpose, an approach developed by Vatne et al. (1996) to predict the evolution of $N_{i}$ based on experimental investigations on the nature and evolution of the respective nucleation sites has been incorporated. In combination with the present approach the recrystallization textures of Al-alloys can now be predicted for a wide range of microstructural characteristics and processing parameters.

For the simulation of a series of recrystallization textures of cold rolled samples, the strain $\varepsilon$, the rolling texture, and some parameters to characterize the microstructure prior to the deformation must be known, namely the initial grain size $D_{0}$, the initial fraction of cubeoriented grains $M_{0}$, as well as the parameters $L$ and $H$ or $N_{0}$ that describe the particle distribution (Eq. (6)). When recrystallization textures of samples deformed at elevated temperatures are to be simulated, additionally the processing parameters strain rate and deformation temperature must be considered (Engler and Vatne, 1998). Then, the model constants $c_{\text {cube }}, c_{\mathrm{GB}}$ and $c_{\mathrm{PSN}}$ describing the efficiency of nucleation at the respective nucleation site and - if desired - the growth efficiency $G$ (Eq. (21)) are determined by fitting experimental data. As already stated 
by Vatne et al. (1996), the modeling constants $c_{i}$ turned out to only depend on the alloy. Hence, fitting of one of the experimental recrystallization textures is sufficient to derive the modeling constants and the model is able to predict recrystallization textures within a given range of experimental parameters for this alloy. Finally, the model can be run to perform the rolling texture transformations and to derive the weight factors $x_{i}$ and, therewith, the recrystallization textures can be simulated.

With regard to the different alloys analyzed, it is known that nucleation at grain boundaries is much more pronounced in commercial purity A1 (AA1145) than in Al-Mn and Al-Cu alloys, which shows up in a much stronger R-component in the former alloy. In the model this is reflected in a high value $c_{\mathrm{GB}}=0.8$ but a low value of $c_{\mathrm{PSN}}=0.15$ in AA1145, whereas in the other two alloys the opposite ratio was obtained. However, it is noted that in all cases the constants $c_{i}$ were of the order of 1 , which means that the underlying model assumptions to derive the nucleus numbers $N_{i}$ appear to be reasonable.

Of course, the applicability of any model is restricted by the underlying fundamental assumptions, in the present case the $40^{\circ}\langle 111\rangle$-(micro)growth selection out of a limited spectrum of nucleus orientations. Thus recrystallization textures forming through other mechanisms, e.g. continuous (in situ) recrystallization or dynamic recrystallization, cannot be simulated. As already mentioned above, the influence of the annealing temperature, which is mainly due to precipitation effects at certain recrystallization temperatures, is also not considered in the model so far. With regard to a possible extension of the model beyond the current cases, i.e. simulation of the recrystallization textures of rolled Al-alloys, again the basic assumptions of the model must be considered. However, even if it turned out that in the case of other materials or deformation modes the assumption of growth selection out of a limited nucleus spectrum were well fulfilled, the computation of the weight factors $x_{i}$ had to be adapted, and for that as detailed microstructural investigations on the nucleation mechanisms as in the present case of recrystallization of rolled Al-alloys are required.

\section{Acknowledgements}

The author would like to thank Dr. H.E. Vatne (Hydro Aluminium Research Centre, Norway) and Dr. U.F. Kocks (Los Alamos Nat. Lab.) 
for helpful discussions. Financial support given by the U.S. Department of Energy and by the Alexander von Humboldt-Foundation through a Feodor Lynen Research Fellowship is gratefully acknowledged.

\section{References}

Bellier, S.P. and Doherty, R.D. (1977). Acta metall. 25, 521-538.

Bunge, H.J. (1982). Texture Analysis in Materials Science, Butterworths, London.

Doherty, R.D. (1978). In Recrystallization of Metallic Materials, edited by F. Haeßner, Dr. Riederer Verlag, Stuttgart, pp. 23-61.

Doherty, R.D., Samajdar, I., Necker, C.T., Vatne, H.E. and Nes, E. (1995). In Proc. 16th Risø Int. Symp., edited by N. Hansen et al., Risø Nat. Lab., Roskilde, 1-23.

Engler, O. (1995). Textures and Microstructures 23, 61-86.

Engler, O. (1996). Mat. Sci. Tech. 12, 859-872.

Engler, O. (1997a). Textures and Microstructures 28, 197-209.

Engler, O. (1997b). Scripta Mater. 37, 1675-1683.

Engler, O. (1998). Acta Mater. 46, 1555-1568.

Engler, O. and Vatne, H.E. (1998). JOM 50, No. 6, $23-27$.

Engler, O., Kong, X.W. and Yang, P. (1997). Scripta Mater. 37, 1665-1674.

Engler, O., Vatne, H.E. and Nes, E. (1996a). Mat. Sci. Eng. A205, 187-198.

Engler, O., Yang, P. and Kong, X.W. (1996b). Acta metall. mater. 44, 3349-3369.

Hjelen, J., Ørsund, R. and Nes, E. (1991). Acta metall. mater. 39, 1377-1404.

Humphreys, F.J. (1977). Acta Metall. 25, 1323-1344.

Humphreys, F.J. (1979). Acta Metall. 27, 1801-1814.

Juul Jensen, D. (1995). Acta Metall. Mater. 43, 4117-4129.

Juul Jensen, D., Hansen, N. and Humphreys, F.J. (1985). Acta Metall. 33, 2155-2162.

Lücke, K. and Engler, O. (1990). Mat. Sci. Tech. 6, 1113-1130.

Nes, E. and Hutchinson, W.B. (1989). In Proc. 10th Risø Int. Symp., ed. J.B. Bilde-Sørensen et al., Risø Nat. Lab., Roskilde, 233-249.

Oscarsson, A. (1991). Textures and Microstructures 14-18, 477-482.

Russell, K.C. and Ashby, M.F. (1970). Acta Metall. 18, 891-901.

Vatne, H.E., Furu, T., Ørsund, R. and Nes, E. (1996). Acta Mater. 44, 4463-4473.

Weiland, H., Rouns, T.N. and Liu, J. (1994). Z. Metallk. 85, 592-597.

Weiland, H. and Hirsch, J. (1991). Textures and Microstructures 14-18, 647-652. 